\title{
The Nuclear Compartment Commonality Hypothesis, Enucleation and the Evolution of the Bacteria and Eukarya
}

James T Staley*

Department of Microbiology and Astrobiology Program, University of Washington, Seattle, WA 98195, USA

\begin{abstract}
The nuclear compartment commonality (NuCom) hypothesis posits that the Bacteria Domain and EukaryaArchaea Domains share a major commonality in the process of DNA replication. The ancestors of both types of organisms used a similar or perhaps identical nuclear envelope platform and nuclear compartment to carry out DNA replication. The effects of this are still seen in the Bacteria and Eukarya all of which have retained membranes with ester-linked, fatty acid lipids. These genes were lost in a process of reductive evolution, termed Enucleation, resulting in the formation of the Archaea from the Eukarya. Some of the nucleated Bacterial phyla such as the Planctomycetes and Verrucomicrobia likely underwent Enucleation giving rise to the more typical Bacterial phyla that lack nuclei. For example, the Verrucomicrobia may be ancestral to the Proteobacteria. The NuCom hypothesis also provides an alternative explanation to opposing views regarding the importance of the Bacteria in the evolution of the Eukarya, namely that these two Domains share a hereditary commonality in their original nuclear envelopes.
\end{abstract}

Keywords: Evolution of bacteria and eukarya; Enucleation

\section{Introduction}

The origin of the Three Domains, the Bacteria, Eukarya and Archaea remains one of the most perplexing problems of understanding biological evolution. How is it that some basic features of the Bacteria, such as their ester-linked lipids and associated membrane features, are also found in the Eukarya but not in the Archaea? The nuclear compartment commonality ( $\mathrm{NuCom}$ ) hypothesis proposes a resolution to this outstanding issue.

Current dogma in biology recognizes that two fundamentally different types of organisms evolved with respect to the information processes of translation, transcription and replication. One is found in the Bacteria, the other in the Eukarya and Archaea. Is there anything in common between these two different biological types? The nuclear compartment commonality hypothesis proposes that the two lineages are linked through the process of DNA replication. Of the three major informational processes, replication was the final one that evolved [1]. Replication is also the process in which the Bacteria exhibit the most marked differences from that of the Eukarya and Archaea.

A nuclear envelope consisting of its inner membrane and outer membrane is required for DNA replication in eukaryotic organisms $[2,3]$. The inner membrane contains numerous proteins that are involved in chromatin organization and membrane anchoring [3]. The NuCom hypothesis is in accord with this requirement in that it posits that when replication first evolved, a nuclear compartment with its membrane envelope was essential. A likely explanation for this requirement is that DNA replication needed a membrane scaffold or platform for the assembly and alignment of the substrate nucleic acids for replication and probably for transcription as well. Also, by isolating the precursor RNA and DNA subunits and templates in the nuclear compartment, they were separated from other molecules that might interfere with the process. Therefore, the nuclear envelope and compartment provided an appropriate platform for replication that was efficient and delivered a high degree of fidelity. The nuclear compartment likely began evolving endogenously [4]. The NuCom proposes that this happened early on before the Domains diverged from one another and likely in concert with the process of transcription.
Two different types of RNA templates initially evolved for DNA replication: one for the precellular Bacteria and the other for the precellular Eukarya-Archaea branch of the Tree of Life. The common, unifying aspect is that the nuclear compartment with its envelope served as either the same site or a very similar site for DNA replication in both lineages. As a result, the Bacteria and the Eukarya share the same or very similar ester-linked, fatty acid membranes from the time that the compartmentalized nucleus evolved. These ester-linked, fatty acid membranes include those of the nuclear compartment envelope (both its inner and outer membranes with their associated proteins [3]), the endoplasmic reticulum and the cell membrane.

\section{Evidence in Support of the NuCom Hypothesis}

If the NuCom hypothesis is true, it implies that the nuclear compartment evolved very early in evolution, a view that is consistent with that of others [5-7]. If so, which representatives of the two primary lineages, the Eukarya-Archaea and the Bacteria, were the first to evolve DNA replication?

The complex nature of the cells of the Eukarya with their distinctive nucleus has been known even before the advent of the electron microscope, hence their name means 'true nucleus.' Therefore, it is literally the sine qua non, or definition of the Eukarya. Thus, the Eukaryotic lineage evolved from a nucleated predecessor long before the lineage speciated.

For the Bacteria, evidence in support of the NuCom hypothesis about the early evolution of the nucleus comes primarily from

*Corresponding author: James T Staley, Department of Microbiology and Astrobiology Program, University of Washington, Seattle, WA 98195, USA, E-mail: jtstaley@u.washington.edu

Received September 18, 2013; Accepted October 28, 2013; Published October 31,2013

Citation: Staley JT (2013) The Nuclear Compartment Commonality Hypothesis, Enucleation and the Evolution of the Bacteria and Eukarya. Astrobiol Outreach 1: 105. doi: $10.4172 / 2332-2519.1000105$

Copyright: (C) 2013 Staley JT. This is an open-access article distributed under the terms of the Creative Commons Attribution License, which permits unrestricted use, distribution, and reproduction in any medium, provided the original author and source are credited. 
independent studies of cell fine structure, cell composition and phylogeny. The most likely candidate for the Bacteria is an early member of the PVC Superphylum (the Planctomycetes - Verrucomicrobia Chlamydia phyla) of the Bacteria, which also includes several other phyla (Poribacter, Lentisphaerae, and the OP3 candidate phylum) not all of which have cultured bacterial representatives. These are likely candidates because some, perhaps all, members of this group have compartmentalized cells with a nucleus $[8,9]$.

Fuerst and Webb [8] were the first to report that members of the Planctomycetes phylum, in particular the species Gemmata obscuriglobus, have a nuclear compartment that is comparable to that of the Eukarya. Their fine structure analyses using cell freeze fracture demonstrated that, like that of the Eukarya, the nucleus is bound by both an outer membrane and inner membrane possibly with nuclear pores [10]. Subsequently other members of the PVC Superphylum of bacteria, including the genus Prosthecobacter in the Verrucomicrobia phylum have also been found that have nuclear compartments [9]. In addition, there is evidence of eukaryotic signature proteins in Gemmata sp. Wa-1 and Prosthecobacter dejongeii including those associated with the nuclear membrane [11].

What is the evidence that the nuclear compartment is very ancient? Phylogenetic evidence supports the early divergence of the Bacteria Domain from the Eukarya - Archaea Domains at the deepest branch of the Tree of Life [12]. At that time, the Bacteria branched off from the Tree of Life and began to speciate by Darwinian evolution [12,13]. This proposal contends that members of the PVC phyla were the earliest members of the Bacteria. Since they have a nuclear compartment, this means that the nuclear compartment must have evolved before the Bacteria branched off from the Protoeukarya - Protoarchaea. Therefore, the Protoeukarya also had access to the nuclear compartment which they, too, required for DNA replication.

This does not mean that the Eukarya were very fully evolved or even close to it at that time. Clearly hundreds of millions of years would need to elapse before the Protoeukarya began to evolve by Darwinian evolution. They required an extensive period of time to evolve phagocytosis, entrain the mitochondrion and evolve mitosis, meiosis and sexuality. However, because the Protoeukarya required a nuclear compartment with its envelope in order to carry out replication, this feature had already evolved at or before the time that the Bacteria branched off from the Tree of Life. The view that the Eukarya had ancient distinctive features early on is consistent with that of Woese $[12,13]$.

The question arises, because it is not obvious from the Tree of Life, What is the evidence that the PVC Superphylum is an ancient group? First of all, it should be noted that in the analysis of deep branches, the resolution of $16 \mathrm{~S}$ rRNA sequences is inadequate to identify lineages with certitude, so this may explain this aberration in the Tree of Life. Nonetheless, there are two primary lines of evidence in support of an ancient lineage for the PVC organisms based on phylogeny. First, Brochier and Phillipe [14] conducted an analysis of the most highly conserved regions of the $16 \mathrm{~S}$ rRNA gene sequences of Bacteria and from that careful analysis concluded that the Planctomycetes phylum was the deepest branch of the Bacteria.

Another piece of phylogenetic evidence comes from studies of the $\mathrm{C}_{1}$ transfer genes involved in the metabolism of one carbon compounds. Their gene products carry out the oxidation of formaldehyde and the reduction of formate. Quayle and Ferenci [15] proposed that the metabolism of one carbon compounds, such as formaldehyde, likely evolved very early on because of their abundance and high chemical reactivity. The oxidation of methane to carbon dioxide is carried out by methanotrophic members of the Bacteria. And the reduction of carbon dioxide to methane occurs by methanogenic members of the Archaea. Both processes use the $\mathrm{C}_{1}$ transfer gene products to mediate reactions at the oxidation level between formaldehyde and formate, but in reverse directions. Phylogenetic analyses for six of the derived proteins (i.e. enzymes) for these reactions all gave the same result [16]: all Planctomycetes members that were analyzed occupied an intermediate position between members of the aerobic Proteobacterial methanotrophs which are true Bacteria and members of the anaerobic methanogenic Archaea. These results not only imply that the Planctomycetes phylum and its close relatives comprised the first organisms in which these processes evolved but provide further evidence of their deep lineage. Indeed, since this author believes inter-Domain horizontal gene transfer events may be questionable, this could be interpreted as a process that evolved before the Bacteria branched off the TofL by a protobacterial ancestor of the nascent PVC phyla. Curiously, although the roles of the enzymes for methanotrophy and methanogenesis are well known, the nature and role of the gene products for the PVC group still remain unknown.

Ancillary support for the early origin of the PVC group is the discovery of an acidophilic methanotrophic species of the Verrucomicrobia, Acidomethylosilex fumarolicum [17]. This bacterium was isolated from $50 \mathrm{C}$ enrichments from acidic $(\mathrm{pH} \mathrm{1)} \mathrm{hot}$ springs indicating this geochemically important process is also found in the PVC phyla. Although this species is an aerobe, evidence has been reported of phylogenetically related methane oxidizing enzymes in a consortium of unidentified bacteria responsible for anaerobic methane oxidation which obtains oxygen from nitrites [18]. Therefore, it is reasonable that this was an important early process of energy generation during the anoxic period on early Earth because methane was abundant then and nitrogen oxides were likely present, too [1820].

Also noteworthy in this regard is that very few phyla of the Bacteria contain sterols in their membranes. Those known to contain sterols include the Planctomycetes [21] and the methanotrophic Proteobacteria. Sterols are found elsewhere only in membranes of the Eukarya which suggests that membrane sterols may be a critical component of the nuclear membrane compartment for both genetic lineages.

Another line of evidence that supports the commonality between the PVC phyla and the Eukarya comes from membrane coat (MC) proteins. They occur in all eukaryotic membranes where they are involved in conferring shape to the membranes. Likewise, they have been found in membranes of the Planctomycetes [22]. These investigators conclude their results indicate that the PVC superphylum contributed to eukaryogenesis. This author infers that this evidence provides support for a common nuclear compartment heritage between the PVC bacteria and the Eukarya as proposed by the NuCom hypothesis.

Therefore, cell structural, compositional and in particular, phylogenetic evidence strongly support the view that the PVC Superphylum is an ancient group at the ancestral base of the Bacteria. One interpretation of this evidence is that the PVC lineage likely gave rise to other phyla of the true Bacteria by a type of reductive evolution that produced true prokaryotic organisms with a simplified system of 
replication and cell structure. In a similar fashion, the Eukarya nuclear membrane evolved early on, probably hundreds of millions of years before the Eukarya began to speciate.

The Eukaryal lineage (as Protoeukarya and Protoarchaea) evolved independently after the Bacteria branched off from the Tree of Life [12,23]. Like the Bacteria, the Protoeukarya lineage retained the compartmentalized nucleus. At this time there are no reports of Archaea that have a nuclear compartment that is analogous to that of the PVC group although Ignicococcus hospitalis, a member of the Crenarchaeota, has two membranes, the outer one of which is involved in ATP generation [24,25]. Perhaps the Eukarya were the only nucleated members of the Eukarya - Archaea branch.

Notably, the Archaea are separated into two phylogenetic groups, the Euryarchaeota and the Crenarchaeota. Those who have studied the Archaea regard relatives of the Crenarchaeota, the TACK Superphylum (Thaumarchaeota, "Aigarchaeota", Crenarchaeota and Korarchaeota phyla) as a 'sister' group of the Eukarya [26-28] because they share many genes with the Eukarya. For example, their DNA replication machinery is strikingly similar to that of the Eukarya [29]. They use some of the same DNA polymerases for DNA replication that are used by the Eukarya, not the DNA polymerase, polD, of the Euryarchaeota.

\section{Enucleation}

With the exception of the PVC group, the other bacterial lineages are typical prokaryotic organisms whose DNA is not compartmentalized. These descendant lineages likely evolved from an early member of the PVC group through a type of reductive evolution. This probably happened soon after the earliest PVC members evolved. Since this reductive process involved the loss of the nucleus, it is referred to as Enucleation (see Glossary). Enucleation resulted in a marked departure from the previous evolutionary trajectory of the PVC bacteria and their typical prokaryotic bacterial offspring. For example, the prokaryotic descendants likely had an accelerated rate of evolution because they were simpler organisms with high growth rates. Therefore Enucleation may have introduced a confounding effect on evolutionary processes that rely on the conserved genes and proteins of single lineages.

An example of a typical bacterial phylum that may have descended from the Verrucomicrobia of the nucleated PVC Superphylum, is the Proteobacteria with its five classes [30], the Alpha-, Beta, Gamma-, Delta- and Epsilon proteobacteria. Some remarkable and several unique features are found among members of both the Verrucomicrobia and the Proteobacteria including budding cell division, prostheca (cellular appendage) formation (as in the genera Verrucomicrobium and Prosthecobacter of the Verrucomicrobia and Caulobacter, Hyphomicrobium and Prosthecomicrobium of the Alphaproteobacteria), methanotrophy, internal cell membranes (e.g., photosynthetic membranes of the Proteobacteria) and sterols. However, apart from the study of the $\mathrm{C}_{1}$ genes by Chistoserdova et al. [16], there have been no other significant phylogenetic linkages reported of which this author is aware to substantiate this proposed relatedness.

The Archaea phyla with their isoprenoid membranes seem to have evolved somewhat differently than simply through a process of reductive evolution from the Eukarya. The origin of the isoprenoid membranes that are typical of the Archaea remains an open question that is not addressed by the NuCom hypothesis but has been proposed to have happened through the complementary processes of thermoreduction and r-selection [5]. In any event, it is also an example of an Enucleation process that resulted in the loss of the nucleus.

\section{Conclusion}

Genomics scientists who have studied the evolution of the Eukarya have reached an impasse [31]. Some argue that the Bacteria played a role in the evolution of the Eukarya [22] whereas others counter that the Eukarya are a sister group of the Archaea and that any relatedness to the Bacteria is due to analogy [32]. The nuclear compartment commonality ( $\mathrm{NuCom}$ ) hypothesis provides an alternative explanation to these two opposing views, namely that both groups evolved from nucleated ancestors that required a similar nuclear envelope during the time that DNA replication evolved.

The nuclear compartment commonality hypothesis explains one of the most basic and enduring puzzles of evolutionary biology: Why do the Bacteria and Eukarya share the same ester-linked lipid membranes? Most scientists have previously proposed alternative hypotheses to explain this purported anomaly. Several have posited that the Eukarya ester-linked membranes came from a 'fusion' event between two different organisms or their nuclei [33]. One was an archaeon, the other a bacterium. The bacterial partner was regarded as the source of the ester-linked fatty acid membranes. The $\mathrm{Nu}$-Com hypothesis is very different and does not require a fusion event between two distinctly different organismal types.

The Nu-Com proposal contends that the ester-linked, fatty acid membranes of the Bacteria and Eukarya are similar to one another because they share a common and very ancient, pre-cellular heritage of their nucleus. Further, these membranes came from the same precellular pool of nuclear compartment membranes replete with sterols and MC proteins. It is important to recognize that early on the Protoeukarya was an immature, still evolving lineage that, in contrast to the Bacteria and Archaea, would have left little trace in the fossil record until approximately $2.5 \mathrm{Ga}$ bp [34]. There are several possible reasons for this: they did not produce any detectable distinctive evolutionary biomarkers or metabolic products, such as the oxygen of the cyanobacteria or the methane of the methanogens; they likely eked out a living with a limited metabolic capability so that they were not able to thrive and become abundant in most environments, at least until they developed phagocytosis when that they could ingest members of the Bacteria; their cells were not bound by a cell envelope with distinctive constituents aside from sterols that are also produced by some members of the Bacteria.

The NuCom hypothesis also posits that the nucleus was the first organelle to evolve, indeed it evolved even before organisms began to speciate. This may seem peculiar to those who have thought that it is of much more recent origin due to its current complexity. Of course, the earliest Protoeukaryal nuclei were still evolving at that early time when the Bacteria began to speciate, because much more evolution and hence, time, was required for the Protoeukarya to perfect the more complex processes of phagocytosis, mitosis, meiosis and sexuality. The likelihood that the early eukaryotic nucleus was still very immature is consistent with that of Devos et al. [4] and is strengthened by recognizing that the nuclei of the PVC group that originated early on have relatively small genomes with a single chromosome in comparison with that of typical modern Eukaryotic organisms. Also, the Archaea, which also have small genomes, probably began diverging from the Eukarya during the Protoeukaryal stage well before the Eukarya began to speciate. 
A variety of proposals have been put forward to explain how the nucleus may have evolved, almost all again involving the fusion of a bacterial cell or genome with that of an archaeon or its genome, with or without endosymbiosis [35-37]. Devos et al. [4] have proposed an endogenous formation, which this author favors. Moreover, this author proposes that it evolved very early on in that DNA replication was a central key to life's processes by providing the proper organization and reliable replication of genetic material. One could argue that the earliest protoeukaryotes were simple ester membrane - bound protocells with simple nuclei that could replicate and use an as yet unknown mechanism for ATP generation during the early long period of Earth's anoxia.

The process of Enucleation, which is as an example of extreme reductionism in which the entire nucleus is lost, remains a mystery. It is not termed 'denucleation' because that implies the nucleus was ejected from a nucleated ancestor leaving behind a prokaryote, which seems highly improbable. Perhaps the process proceeded through a type of streamlining in which genes that were unnecessary for the habitation of a particular niche were lost over time. Alternatively, a disgorgement or spawning mechanism may have occurred in which the nucleated cell formed a cell from within, much like that of an organelle, which contained a minimal set of genes for survival. This 'organelle' may have been released by a budding process, the characteristic form of cell division in some members of the PVC group such as the Plantomycetes. Alternatively, it could have escaped through the lysis of the nucleated "host" by a virus or another lytic event.

Interestingly, although the NuCom hypothesis was formulated independently, it is consistent with others [5-7] who explain the origin of the Domains from a complex LUCA (Last Universal Common Ancestor). Their hypotheses also involve the evolution of prokaryotic organisms, the Bacteria and Archaea through reductionist mechanisms from LUCA. Therefore their independently conceived views add further support for the NuCom hypothesis.

This hypothesis accounts for many of the major similarities found in the Eukarya that are also found in the Bacteria. However, other genes are shared between these two separate Domains including homologs for eukaryotic $\alpha$ - and $\beta$ - tubulin which are also found in the PVC genus Prosthecobacter where they are named BtubA and BtubB [38]. Interestingly, although some dismiss these homologs as being examples of a horizontal gene transfer from the Eukarya to Prosthecobacter $[32,39]$ recent evidence indicates these are of very ancient origin $[40,41]$. The ancient heritage of BtubA and Btub B is also consistent with the view expressed by Carl Woese [13].

Other genes shared between the Bacteria and Eukarya, apart from those genes received from the mitochondrion [42] may date back to the period before the Domains separated, prior to the time the Bacteria branched off from the Tree of Life.

There are also examples of traits that are found in the Archaea and Bacteria that are not found in the Eukarya. These include the $\mathrm{C}_{1}$ genes for the metabolism of one carbon compounds [16]. In addition, the cell envelope peptidoglycan of the Bacteria and pseudo-peptidoglycan found in the Euryarchaeota, are other examples of similarities between these groups. In this regard it is of interest to note that those phyla that are most closely related to the nuclear core phylogenetically, such as the TACK superphylum and the PVC superphylum either lack the genes for peptidoglycan synthesis altogether or have a partial set of them. Likewise, FtsZ, like peptidoglycan is found in the more recently evolved phyla of the Bacteria and the Archaea, but not in the TACK superphylum and only some members of the PVC superphylum of the Bacteria. Occasionally peptidoglycan and FtsZ genes have been found in Eukaryotes and has been explained by the acquisition of bacterial endosymbionts [32]. The explanation for the occurrence of the peptidoglycan and FtsZ genes in the Bacteria and Archaea is still debated and remains to be resolved.

Genes that are sufficiently small may have been transferred between the Bacteria and Archaea via a virus or even by transformation. For example, those for gas vesicle synthesis are comprised principally of the genes for two proteins, the major one, GvpA, which consists of 70 amino acid residues [43]. These likely evolved early on which is consistent with the proposal that gas vesicles may have been responsible for the earliest form of cellular motility in Bacteria and Archaea [44].

Finally, one of the most important consequences of one aspect of the NuCom hypothesis is that nucleated organisms evolved first, before prokaryotic organisms arose from them. For both the PVC Bacteria and especially the Eukarya it seems reasonable that the prokaryotes branched off during the evolution of the lineages, before the time they had fully evolved and speciated. If true, the enucleate members of the Bacteria and the Archaea should be more appropriately referred to as Postkaryotes rather than Prokaryotes as they were derived from the reductive evolution of nucleated ancestors, a proposal that is consistent with that of Poole et al. [5], Kurland et al. [6] and Glansdorff et al. [7].

The NuCom hypothesis raises a number of questions that may be addressed experimentally. Hopefully, these and other issues will be the subject of further study to test the validity of the NuCom hypothesis.

\section{Acknowledgement}

I am very grateful to Frank Harold who gave important advice through his supportive criticisms of NuCom, his comprehension of the growing literature in this area, and our enjoyable discussions of the hypothesis. William Whitman provided several important suggestions that were very helpful in rewriting this paper. In addition, Roger Buick provided valuable insight particularly on the most appropriate geological references. Evgeni Sokurenko made several useful suggestions, especially on the organization of the paper.

\section{References}

1. Olsen GJ, Woese CR (1996) Lessons from an archaeal genome: what are we learning from Methanococcus jannaschii? Trends Genet 12: 377-379.

2. Laskey R, Medine M (1996) DNA Replication in Eukaryotic Cells, Cold Spring Harbor Press.

3. Hetzer MW (2010) The nuclear envelope, Perspectives in Biology, Cold Spring Harbor Press.

4. Damien Devos, Svetlana Dokudovskaya, Frank Alber, Rosemary Williams, Brian T Chait, et al. (2004) Components of coated vesicles and nuclear pore complexes share a common molecular architecture. PLoS Biol 2: 2085-2093.

5. Poole A, Jeffares D, Penny D (1999) Early evolution: prokaryotes, the new kids on the block. BioEssays 21: 880-889.

6. Kurland CG, Collins LJ, Penny D (2006) Genomics and the irreducible nature of eukaryotic cells. Science 312: 1011-1014.

7. Glansdorff N, Xu Y, Labedan B (2008) The last universal common ancestor: emergence, constitution and genetic legacy of an elusive forerunner. Biology Direct 3: 29 .

8. Fuerst JA, Webb RI (1991) Membrane-bounded nucleoid in the eubacterium Gemmata obscuriglobus. Proc Natl Acad Sci U S A 88: 8184-8188.

9. Lee KC, Webb RI, Janssen PH, Sangwan P, Romeo T, et al. (2009) Phylum Verrucomicrobia representatives share a compartmentalized cell plan with members of bacterial phylum Planctomycetes. BMC Microbiol 9: 5.

10. Fuerst JA (2005) Intrcellular compartmentation in Planctomycetes. Ann Rev Microbiol 59: 299-328. 
Citation: Staley JT (2013) The Nuclear Compartment Commonality Hypothesis, Enucleation and the Evolution of the Bacteria and Eukarya. Astrobiol Outreach 1: 105. doi: 10.4172/2332-2519.1000105

Page 5 of 5

11. Staley JT, Bouzek $H$, Jenkins $C$ (2005) Eukaryotic signature proteins of Prosthecobacter dejongeii and Gemmata Wa-1. FEMS Microbiol Lett 243: 9-14.

12. Woese CR (2002) On the evolution of cells. Proc Natl Acad Sci U S A 99 $8742-8747$.

13. Woese CR (2004) A new biology for a new century. Microbiol Mol Bio Rev 65 173-186.

14. Brochier C, Philippe H (2002) Phylogeny: a non-hyperthermophilic ancestor for bacteria. Nature 417: 244.

15. Quayle JR, Ferenci T (1978) Evolutionary aspects of autotrophy. Microbiol Rev 42: 251-273.

16. Chistoserdova L, Cheryl J, Marina GK, Christopher JM, Alla L, et al. (2004) The enigmatic Planctomycetes may hold a key to the origins of methanogenesis and methylotrophy. Mol Biol Evol 21: 1234-1241.

17. Pol AK, Heijmans HR, Harhangi DT, MSN Jetten, den Camp, et al. (2007) Methanotrophy below $\mathrm{pH} 1$ by a new Verrucomicrobia species. Nature Lett 450.

18. Katharina FE, Margaret KB, Denis LP, Eric P, Sophie M, et al. (2010) Nitritedriven anaerobic methane oxidation by oxygenic bacteria. Nature 464: 543

19. Garvin J, Buick R, Anbar AD, Arnold GL, Kaufman AJ (2009) Isotopic evidence for an aerobic nitrogen cycle in the late Archean. Science 323: 1045-1048.

20. Rachel SM, Andreas J, Matyas G, Ulrike B, Aidan B, et al. (2010) The compartmentalized bacteria of the Planctomycetes-VerrucomicrobiaChlamydiae Superphylum have membrane coat-like proteins PLoS Biol. 8: 1-11

21. Godfrey LV, Falkowski PG (2009) The cycling and redox state of nitrogen in the Archaean ocean. Nature Geosci 2: 725-729.

22. Pearson A, Budin M, Brocks JJ (2003) Phylogenetic and biochemical evidence for sterol synthesis in the bacterium Gemmata obscuriglobus. Proc Natl Acad Sci U S A 100: 15352-15357.

23. Kandler O (1994) Cell wall chemistry of Archaea and its phylogenetic implications. J Biol Phys 20: 165-169

24. Rachel R, Wyschkony I, Riehl S, Huber H (2002) The ultrastructure of Ignicococcus: Evidence for a novel outer membrane and for intracellular vesicle budding in an archaeon. Archaea 1: 9-18.

25. Küper U, Meyer C, Müller V, Rachel R, Huber H (2004) Energized outer membrane and spatial separation of metabolic processes in the hyperthermophilic Archaeon Ignicoccus hospitalis Proc Natl Acad Sci U S A 107: $3152-3156$

26. Williams TA, Foster AG, Nye TM, Cox CJ, Embley TM (2012) A congruent phylogenomic signal places eukaryotes within the Archaea. Proc Roy Soc London, Series B 279: 4870-4879.
27. Yutin N, Makarova KS, Mekhedov SL, Wolf YI, Koonin EV (2008) The deep archaeal roots of eukaryotes. Mol Biol Evol 25: 1619-1630.

28. Guy L, Ettema TJG (2011) The archaeal "TACK" superphylum and the origin of eukaryotes. Trends Microbiol 19: 580-587.

29. Barry ER, Bell SD (2006) DNA replication in the Archaea. Microbiol Mol Biol Rev 70: 876-887.

30. Bergey's Manual of Systematic Bacteriology (2005) $2^{\text {nd }}$ edn, Volumes $2 A, 2 B$ 2C, Garrity G, Krieg N, Brenner D and Staley JT (eds) Springer, New York.

31. Reynaud EG, Devos DP (2011) Transitional forms between the three domains of life and evolutionary implications. Proc Biol Sci 278: 3321-3328.

32. James OM, William FM, Eugene VK, John FA, Michael YG, et al. (2011) Planctomycetes and eukaryotes: a case of analogy not homology. Bioessays 33: $810-817$

33. Rivera MC, Lake JA (2004) The ring of life provides evidence for a genome fusion origin of eukaryotes. Nature 431: 152-155.

34. George SC, Volk H, Dutkiewicz A, Ridley J, Buick R (2008) Preservation of hydrocarbons and biomarkers in oil trapped inside fluid inclusions for $>2$ billion years. Geochim Cosmochim Acta 72: 844-870.

35. Martin W (2005) Archaebacteria (Archaea) and the origin of the eukaryotic nucleus Current Opin Microbiol 8: 630-637.

36. Martin W, Müller M (1998) The hydrogen hypothesis for the first eukaryote Nature 342: 37-41.

37. Martin W, Koonin EV (2006) Introns and the origin of nucleus-cytosol compartmentation Nature 440: 41-45.

38. Cheryl J, Ram S, lain A, Brian PH, Giulio P, et al. (2002) Genes for the cytoskeletal protein tubulin in the bacterial genus Prosthecobacter. Proc Nat Acad Sci U S A 99: 17049-17054.

39. Schlieper DM, Oliva A, Andreu JM, Lowe J (2005) Structure of bacterial tubulin BtubA/B evidence for horizontal gene transfer. Proc Natl Acad Sci U S A 102 9170-9175.

40. Pilhofer M, Ladinsky MS, McDowall AW, Petroni G, Jensen GJ (2011) Microtubules in bacteria: ancient tubulins build a five-protofilament homolog of the eukaryotic cytoskeleton. PLoS Biology 9: 12

41. Antonio JM, María AO, Laura S, Anamitra B, Marina S, et al. (2011) Bacteria tubulin distinct loop sequences and primitive assembly properties support its origin from a eukaryotic tubulin ancestor. J Biol Chem 286: 19789-19803.

42. Kurland CG, Andersson SGE (2000) Origin and evolution of the mitochondrial proteome. Microbiol Mol Biol Rev 64: 786-820.

43. Walsby AE (1994) Gas vesicles. Microbiol Rev 58: 94-144.

44. Staley JT (1980) The gas vacuole: An early organelle of prokaryote motility? Origins of Life 10: 111-116. 